\title{
BRIEF REPORT Trimming Long-Term Tunneled Central Venous Catheters in Pediatric Patients
}

\author{
Alessandro Inserra, MD, PhD, ${ }^{1}$ Alessandro Crocoli, MD, ${ }^{1 *}$ Andrea Conforti, MD, ${ }^{2}$ \\ Laura De Petris, $\mathrm{MD}^{3}$ and Alessandro Jenkner, $\mathrm{MD}^{4}$
}

Long-term tunneled central venous catheters (CVC) are employed in critically ill patients. Manufacturers do not provide patient-customized devices; therefore, trimming is required for pediatric use. Scanning Electron Microscopy (SEM) coupled with energy-dispersive X-ray spectroscopy and attenuated total reflectionFourier transform infrared spectroscopy (ATR-FTIR) was used to assess changes induced by different trimming methods on single and double lumen Hickman-Broviac catheters. Increased roughness, exposure of inorganic macroaggreagates and increase in surface inorganic charges were generated by the trimming procedure, with the scalpel producing a smoother surface compared to scissors. Trimming produces changes on the CVC surface that may influence the rate of long-term complications. Pediatr Blood Cancer 2013;60:152-155. () 2012 Wiley Periodicals, Inc.

Key words: bacterial adhesion; central venous catheters; thrombosis; trimming

\section{INTRODUCTION}

Long-term central venous catheters (CVC) constitute a mainstay in the management of critically ill children providing safe vascular access for all clinical needs. However, they also carry a risk of complications that may lead to potentially life-threatening conditions [1-5]. Although, thrombosis and bacterial colonization have been well studied [2-5], the consequences of CVC trimming with scalpel or scissors, a common practice in pediatrics, performed in order to adapt length to patient size, have been only rarely reported [6-9]. Furthermore, totally implanted devices (Ports) are trimmed at the proximal end but may have significant drawbacks such as rupture or rotation, bleeding in thrombocytopenic episodes and ulcer formation in thin patients, so that their use in small children is still controversial, even if they are optimal for adolescents requiring intermittent treatment. Moreover, to date no data have been published on the molecular and biochemical surface modifications induced by trimming.

The aim of the study was to assess molecular and ultrastructural changes to the material employed secondary to the trimming procedure and to discuss the implications concerning thrombosis and infections.

\section{CASE}

Twenty-one single and double lumen Hickman-Broviac catheters (single lumen, 9.6 Fr. double lumen 12 Fr. -Bard Access System Inc., Salt Lake City, UT) were immediately processed for electron microscopy upon removal from the commercial packaging. All devices were purchased from commercial sources and the manufacturer was not involved in the study. The catheter was handled with Adson non-toothed forceps and ten transverse sections were obtained using either sterile Mayo scissors (five sections) or scalpel (Granton Silver no. 11 surgical blade, Granton Lagg Ltd, Sheffield, UK; five sections). A total of 210 sections were thus available for analysis. Uncut manufactured tips were used as controls.

Scanning Electron Microscopy (SEM) was used for imaging of all samples, which were mounted on a sputter-coated specimen stub and examined with a LEO-1450 VP microscope (LEO, Oberkochen, Germany) equipped with an X-ray probe.

Two distinct SEM approaches were employed: the configuration based on secondary electrons provided information on surface topography, while that based on backscattered electrons
(BSE) provided information on the atomic number of the average area of origin, topography and crystalline structure.

SEM was also coupled with an energy-dispersive X-ray spectroscopy (EDS) probe (Inca Energy, Oxford Instruments: Tubney Woods, Oxfordshire, UK) to determine the chemical composition of the sample and investigate the distribution of elements within the polymer.

Attenuated total reflection-Fourier transform infrared (ATRFTIR) spectroscopy is a technique that allows recording of ATR spectra directly on samples by means of non-destructive analysis; it was employed to investigate chemical differences on the new surfaces generated. All spectra were recorded on a Spectrometer equipped with Universal ATR Sampling Accessory (Perkin-Elmer Inc., Waltham, MA), using a Diamond Crystal (refractive index $\mathrm{n}=2,419)$ as ATR probe. The infrared spectra were collected within the 650 and $4,000 \mathrm{~cm}^{-1}$ wavelengths with $4 \mathrm{~cm}^{-1}$ resolution.

\section{RESULTS}

SEM findings in all our 210 specimens (105 scalpel, 105 scissor-trimmed) showed trimmed tips characterized by evident spikes and depressions, secondary to the combined action of pressure, cutting and tearing by the blades, which were much less evident in scalpel-trimmed specimens and completely absent in the controls, which displayed a smooth surface (Fig. 1).

BSE photomicrographs showed the appearance of inorganic macroaggregates on the cut surface, which were never evident on native catheters (Fig. 2A-C). These changes may be ascribed

Additional Supporting Information may be found in the online version of this article.

${ }^{1}$ Division of Pediatric Surgery, Bambino Gesù Children's Hospital, Rome, Italy; ${ }^{2}$ Division of Neonatal Surgery, Bambino Gesù Children's Hospital, Rome, Italy; ${ }^{3}$ Division of Nephrology, Mount Sinai School of Medicine, New York, New York; ${ }^{4}$ Division of Pediatric Hematology-Oncology, Bambino Gesù Children's Hospital, Rome, Italy

Conflict of interest: Nothing to declare.

*Correspondence to: Alessandro Crocoli, MD, Division of Pediatric Surgery, Bambino Gesù Children's Hospital, Piazza S. Onofrio 4, 00165 Rome, Italy. E-mail: alessandro.crocoli@opbg.net

Received 20 March 2012; Accepted 9 August 2012 


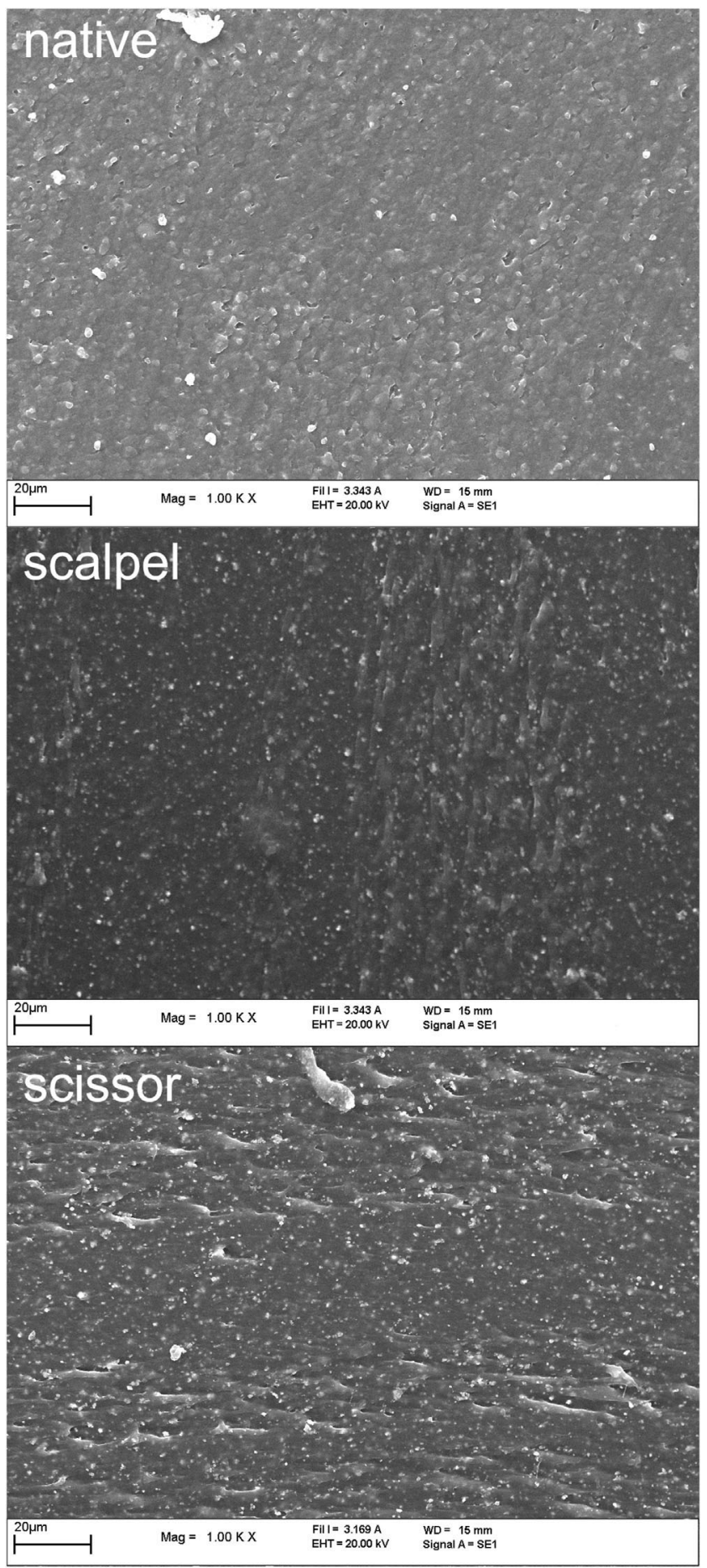

Fig. 1. SEM photomicrograph (SE) of untrimmed (native) and trimmed samples (either scalpel or scissors).

to the removal of the "frozen skin" (a thin polymer layer poor in inorganic charge). The removal of this layer reveals a new surface where macroaggregates, which are normally embedded within the catheter, are exposed.

EDS scanning revealed the presence of barium sulfate $\left(\mathrm{BaSO}_{4}\right.$, a high-density compound exploited for its radio-opaque properties) within the specimens (Supplemental Fig. 1A-C). In Pediatr Blood Cancer DOI 10.1002/pbc particular, Supplemental Figure 1A shows an inorganic charge macroaggregate covered by a thin polymeric film preventing its exposure; the silicon (polymer matrix) and barium (inorganic charge) mappings show that the distribution of these two elements is homogeneous on the sample surface. Supplemental Figure 1B,C show that trimming exposes $\mathrm{BaSO}_{4}$ macroaggregates, which are no longer surrounded by a silicon matrix.

Catheters trimmed with either scissors or scalpel showed similar spectra on ATR-FTIR spectroscopy, but which were only marginally different from those of controls. Specifically, changes were observed at the 1,175 and $1,070 \mathrm{~cm}^{-1}$ absorption peaks which, according to Zhang et al. [10], could be attributed to stretching characteristic absorption peaks of barium sulfate, confirming an increase in surface charge (Supplemental Fig. 1D).

\section{DISCUSSION}

The most common causes of catheter failure are thrombus formation and bacterial colonization [1-5]; however, the molecular and physical interactions that affect bacterial and platelet adhesion to biomaterials have not yet been completely elucidated [6,7]. Jegatheeswaran et al. [9] have clearly demonstrated that trimming is the most important factor involved in tip roughness, and also showed that scissors produce a much rougher surface compared to a scalpel. Surface hydrophobicity and charge appear as additional important factors for both bacterial adhesion and thrombus formation. Both processes are ruled by as yet incompletely understood complex physicochemical interactions. Van Oss and Giese speculated about hydrophobic interactions in biological systems, defining them as "the attraction among apolar or slightly polar cells or other molecules in an aqueous solution" [11]. Bacteria are known to acquire an electrical charge when suspended in water [12], and Oliveira et al. [13] have found a linear correlation between surface hydrophobicity and the number of attached cells, which also applies to the process of bacterial adhesion to indwelling devices. These authors also demonstrated that increased surface roughness exposing an increased amount of inorganic charge facilitates bacterial adhesion and provides a more favorable site for colonization, protecting bacteria from shear forces [14-16]. We have demonstrated that the increased roughness and polarity of the CVC tip, as well as the amount of inorganic charge revealed by SEM and ATR-FTIR analysis, are the direct consequence of trimming. These features, not displayed by native devices, might play a role in both bacterial colonization and thrombus formation [17-20] and directly influence the viability of the device. As previously mentioned, we transected the catheters transversely. The elliptic shape of a beveled tip may actually lead to a high-risk of thrombosis and fibrin sheath formation. We believe that the minimally invasive procedure of ultrasound-guided venipuncture, and subsequent CVC insertion by means of a wire peel-away introducer, does not require trimming of the tip to this shape.

In conclusion, catheter tips constitute the most important site for both bacterial and platelet adhesion and there may be a correlation between the modifications in tip shape induced by trimming and these well-known complications. Clearly only a randomized follow up study would be able to demonstrate that the recorded physical changes do in fact lead to an increase in line complications. 

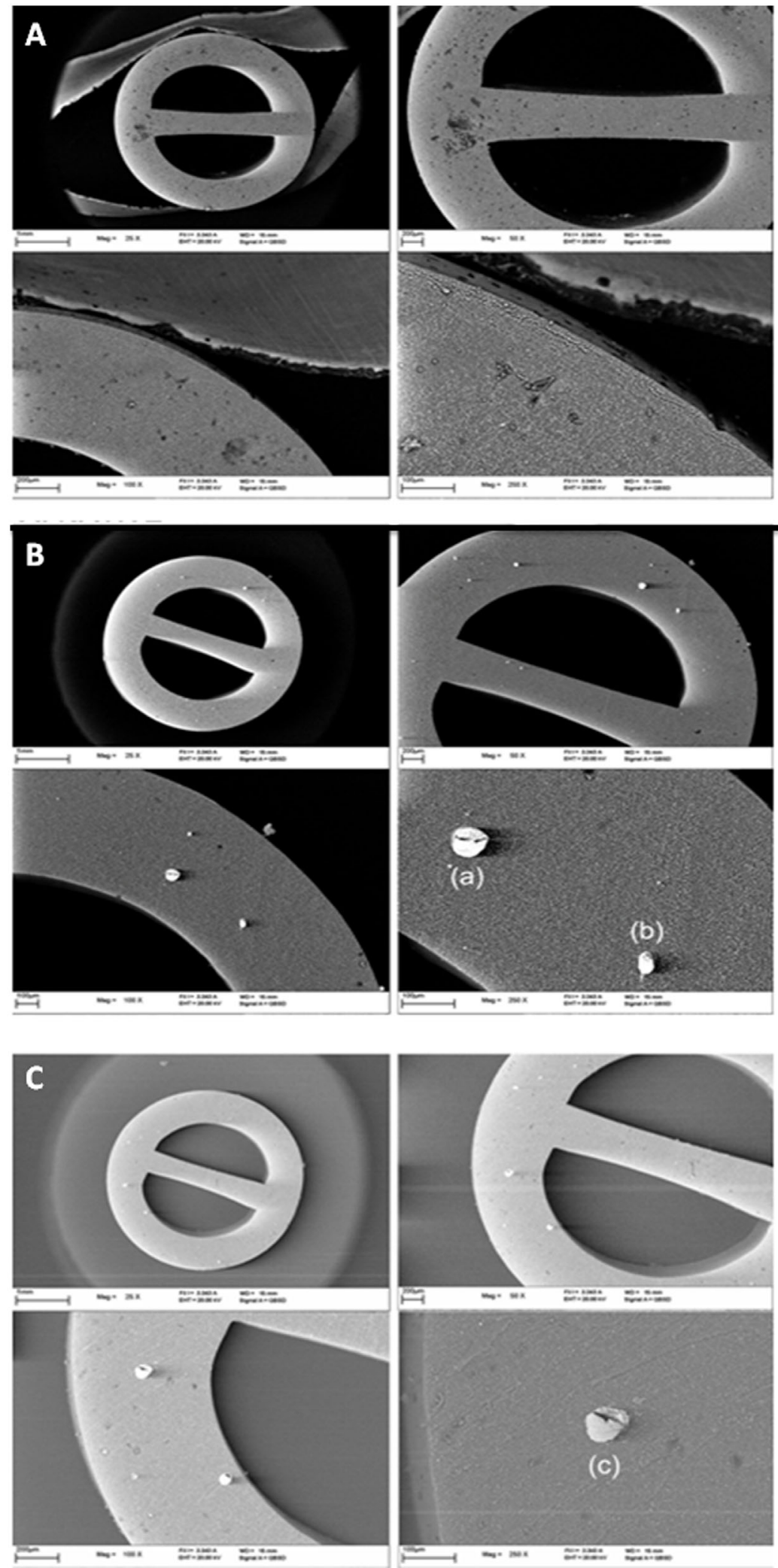

Fig. 2. A: SEM photomicrograph (BSE) of native catheter surface (magnification of 50-2,500×). No evidence of inorganic charge macroaggregates. B: SEM photomicrograph (BSE) of scalpel trimmed catheter surface. Inorganic charge macroaggregates are visible (a, b); C: SEM photomicrograph (BSE) of scissor trimmed catheter surface. Inorganic charge macroaggregates are visible (c). 
With currently available devices, the only way of ensuring a completely smooth distal catheter tip is to use a totally implanted device (Port) in two pieces. As not all patients are suitable for these, we advocate that a Hickman-Broviac device should be designed in two pieces to allow the catheter to be trimmed at its proximal rather than distal extremity.

\section{ACKNOWLEDGMENT}

The authors gratefully acknowledge the contribution of Dr. Alessandro Di Blasio from the Department of Materials Science and Chemical Engineering at the Politecnico di Torino in reviewing the sample analysis, data collection and results.

\section{REFERENCES}

1. Mehall JR, Saltzman DA, Jackson RJ, et al. Fibrin sheath enhances central venous catheter infection. Crit Care Med 2002;30:908-912.

2. O'Grady NP, Alexander M, Dellinger EP, et al. Guidelines for the prevention of intravascular catheterrelated infections. The Hospital Infection Control Practices Advisory Committee, Center for Disease Control and Prevention. US Pediatr 2002;110:e51.

3. Boraks P, Seale J, Price JP, et al. Prevention of central venous catheter associated thrombosis using minidose warfarin in patients with haematological malignancies. Br J Haematol 1998;101:483-486.

4. Massicotte P, Mitchell L. Thromboprophylaxis of central venous lines in children with cancer: The first steps taken on the long road ahead. Acta Paediatr 2006;95:1049-1052.
5. Cesaro $\mathrm{S}$, Corrò $\mathrm{R}$, Pelosin $\mathrm{A}$, et al. A prospective survey on incidence and outcome of Broviac/ Hickman catheter-related complications in pediatric patients affected by hematological and oncological diseases. Ann Hematol 2004;83:183-188. Epub 2003 Nov 13.

6. Sheth NK, Franson TR, Rose HD, et al. Colonization of bacteria on polyvinyl chloride and Teflon intravascular catheters in hospitalized patients. J Clin Microbiol 1983;18:1061-1063.

7. Nachnani GH, Lessin LS, Motomiya T, et al. Scanning electron microscopy of thrombogenesis on vascular catheter surfaces. N Engl J Med 1972;286:139-140.

8. Parvez B, Parmar N, Chan AK. Trimming of peripherally inserted central venous catheters may increase the risk of thrombosis. Thromb Res 2004:113:175-177.

9. Jegatheeswaran A, Parmar N, Walton JM, et al. Quantitative analysis of catheter roughness induced by cutting and manipulation: A potential prothrombotic risk. Blood Coagul Fibrin 2007;18:531-536.

10. Zhang M, Zhang B, Li X, et al. Synthesis and surface properties of submicron barium sulfate particles. Appl Surf Sci 2011;258:24-29.

11. Van Oss CJ, Giese RF. The hydrophilicity and hydrophobicity of clay minerals. Clay Clay Miner 1995;43:474-477.

12. Katsikogianni M, Missirlis YN. Concise review of mechanisms of bacterial adhesion to biomateria surfaces and of techniques used in estimating bacteria-material adhesion. Eur Cells Mater 2004:7737-57.

13. Oliveira R, Azeredo J, Teixeira P, et al. The role of hydrophobicity in bacterial adhesion. In: Gilbert $\mathrm{P}$, Allison D, Brading M, Verran J, Walker J, editors. Biofilm Community Interactions: Chance or Necessity?. Cardiff: Bioline; 2001. pp. 11-22.

14. Sousa C, Teixeira P, Oliveira R. Influence of surface properties on the adhesion of Staphylococcus epidermidis to acrylic and silicone. Int J Biomater 2009; 2009: 718017 [Epub 2009 Jan 25].

15. Chen LT, Phelps CP, Bryant MW, et al. Cellular response to an intravascular catheter. J Submicr Cytol Pathol 2003:35:303-307.

16. An $\mathrm{YH}$, Friedman RJ. Concise review of mechanisms of bacterial adhesion to biomaterial surfaces. J Biomed Mater Res 1998;43:338-348.

17. Kohler TR, Kirkman TR. Central venous catheter failure is induced by injury and can be prevented by stabilizing the catheter tip. J Vasc Surg 1998;28:59-65; discussion 65

18. Guo B, Zhao X, Shi Y, et al. Pathogenic implication of a fibrinogen-binding protein of Staphylococcus Epidermidis in a rat model of intravascular-catheter-associated infection. Infect Immun 2007:75:29912995.

19. Peckham SM, Turitto VT, Glantz J, et al. Hemocompatibility studies of surface-treated polyurethanebased chronic indwelling catheters. J Biomater Sci Polym Ed 1997;8:847-858.

20. Xu LC, Logan BE. Interaction forces between colloids and protein-coated surfaces measured using an atomic force microscope. Environ Sci Technol 2005;39:3592-3600. 